Article

\title{
Early Arterial Intimal Thickening and Plaque Is Related with Treatment Regime and Cardiovascular Disease Risk Factors in Young Adults Following Childhood Hematopoietic Stem Cell Transplantation
}

\author{
Johnny K. M. Sundholm 1,*(D), Anu Suominen ${ }^{2}$, Taisto Sarkola 1,3 ${ }^{\mathbb{D}}$ and Kirsi Jahnukainen ${ }^{2,4}$ \\ 1 Division of Cardiology, Children's Hospital, University of Helsinki and Helsinki University Hospital, \\ 00029 Helsinki, Finland; taisto.sarkola@helsinki.fi \\ 2 Division of Hematology-Oncology and Stem Cell Transplantation, Children's Hospital, \\ University of Helsinki and Helsinki University Hospital, 00029 Helsinki, Finland; \\ anu.suominen@fimnet.fi (A.S.); kirsi.jahnukainen@ki.se (K.J.) \\ 3 Minerva Foundation Institute for Medical Research, 00290 Helsinki, Finland \\ 4 Department of Women's and Children's Health, Karolinska Institute and University Hospital, \\ 17164 Stockholm, Sweden \\ * Correspondence: johnny.sundholm@helsinki.fi
}

Received: 30 June 2020; Accepted: 7 July 2020; Published: 13 July 2020

\begin{abstract}
The long-term vascular effects following childhood hematopoietic stem cell transplantation (HSCT) are not well characterized. We compared arterial wall morphology and function using very-high resolution ultrasound $(25-55 \mathrm{MHz})$ in 62 patients following autologous $(n=19)$ or allogenic $(n=43)$ HSCT for childhood malignancies and hematological disease (median age 25.9 years, IQR 21.1-30.1; median follow-up time 17.5 years IQR 14.1-23.0) with an age matched healthy control group $(n=44)$. Intima-media thickness of carotid (CIMT $0.49 \pm 0.11$ vs. $0.42 \pm 0.06 \mathrm{~mm}, p<0.001$ ), brachial, femoral, radial arteries, and local carotid stiffness, but not adventitial thickness, were increased $(p<0.001)$. Diffuse intimal thickening $(>0.06 \mathrm{~mm})$ of femoral or radial arteries $(n=17)$ and subclinical carotid or femoral plaques $(n=18)$ were more common $(p<0.001)$. Radiation predicted plaques $(p<0.001)$ and local carotid stiffness $(p<0.001)$, but not intimal thickening. CIMT was predicted by age, BMI $>30 \mathrm{~kg} / \mathrm{m}^{2}$, hsCRP $>2.5 \mathrm{mg} / \mathrm{L}$, hypertension, $\mathrm{HbA} 1 \mathrm{c}>42 \mathrm{mmol} / \mathrm{L}$, and cumulative anthracycline $>150 \mathrm{mg} / \mathrm{m}^{2}$. Cumulative metabolic syndrome criteria and cardiovascular disease (CVD) risk factors were more common among HSCT and related with CIMT $(p<0.001)$, but CIMT was similar among controls and HSCT without CVD risk factors. Long-term childhood HSCT survivors show early arterial aging related with radiation, metabolic, and CVD risk factors. Prevention of risk factors could potentially decelerate early arterial wall thickening.
\end{abstract}

Keywords: early vascular ageing; atherosclerosis; radiotherapy; hematopoietic stem cell transplantation; very-high resolution ultrasound

\section{Introduction}

More children are treated with hematopoietic stem cell transplantation (HSCT) for malignant and non-malignant diseases [1]. Survivors are prone to both disease, pretreatment and treatment regime related long term morbidities. Cardiovascular disease (CVD) risk factors are prevalent among childhood cancer survivors treated with HSCT [2-7].

Although the increased prevalence of CVD risk factors following HSCT has been described more thoroughly among adults, there is still lack of information regarding surrogate markers of 
subclinical CVD including arterial stiffness and arterial wall abnormalities in long-term survivors following pediatric HSCT [8]. The interrelations between intima-media thickness (IMT), pulse wave velocity (PWV) and CVD risk factors have not previously been assessed in more detail in this population. The implementation of non-invasive vascular very-high resolution ultrasound (VHRU, 25-55 MHz) has made it possible to investigate the vascular wall in more detail (axial pixel resolution $0.015-0.040 \mathrm{~mm}$ ) [9]. Peripheral arterial vascular wall layer thickness is related with CVD events, but the relationship is not as well established as for carotid IMT and plaque $[10,11]$. We have recently histologically verified the VHRU derived four-line pattern of the arterial far wall as a non-invasive method to assess diffuse intimal thickening simultaneously and separately from medial and adventitial layer thickness [12].

The aims of this study were (1) to assess the prevalence of metabolic CVD risk factors and their surrogate subclinical CVD markers, including arterial layer thickness, plaque, and stiffness, among young adult childhood HSCT survivors with healthy age-matched controls and (2) to assess interrelations between metabolic and CVD risk factors, treatment regimens, and surrogate subclinical CVD markers.

\section{Study Subjects and Methods}

This is a single-center cross-sectional case-control study consisting of two cohorts of HSCT survivors $(n=62)$. In the first cohort, all male survivors treated between 1980 and 2010 at the age of up to 17 years with myeloablative allogeneic HSCT and who on 1 January 2017 were at least 18 years of age were eligible for the study $(n=85)$. Twenty-seven patients had missing contact information or were non-responders, 6 patients declined participation, and 2 patients were excluded as they were unable to give consent. Another 7 subjects were not eligible for the vascular assessment providing a final sample of 43 male subjects. Non-participants did not differ significantly from participants regarding background data.

The second cohort consisted of all high-risk neuroblastoma survivors $(n=23)$ born 1996 or earlier and treated with autologous HSCT in Finland between 1980 and 2000. The cohort also included one high-risk retinoblastoma patient with same treatment regime. Twenty patients consented but one patient died prior to study initiation giving a final sample of 19, the cohort has been further described in Vatanen et al., 2015 [13].

Forty-four healthy controls without history of chronic disease, symptoms or current medication were recruited among hospital personnel, students and teenage children among hospital employees. The controls were age matched as a group with HSCT cases, and the sex distribution between the study groups was similar. The study protocol conforms to the ethical guidelines of the 1975 Declaration of Helsinki, and it was approved by the local ethics board (Helsinki University Hospital, Ethics Committee for gynecology and obstetrics, pediatrics and psychiatry HUS/1742/2016 and HUS 183/13/03/03/2010). Written informed consent was obtained from all participants at enrollment.

Information on diagnosis, treatment, and conditioning regimes was gathered from hospital records including exposure to irradiation (TBI and local testicular and CNS irradiation). Exposure to alkylating agents was calculated as a cumulative cyclophosphamide equivalent dose (CED, mg/m²) [14]. Cumulative exposure to anthracyclines $\left(\mathrm{mg} / \mathrm{m}^{2}\right)$ was calculated with the following conversion factors: Doxorubicin 1.0, Daunomycin 0.833, Idarubicin 5.0, Epirubicin 0.67, and Mitoxantrone 4.0. Acute graft versus host disease (aGVHD) and its gradus were assessed from patient records, whereas chronic GVHD (cGVHD) was prospectively assessed [15].

\subsection{Anthropometrics, Body Composition, Blood Pressure, and Blood Work}

An electronic stadiometer and scale (Seca gmbh \& co. kg, Hamburg, Germany) was used to measure height to the nearest $0.1 \mathrm{~cm}$ and weight to the nearest $0.1 \mathrm{~kg}$ with light clothing and without shoes (Seca 770, Seca gmbh \& co. kg, Hamburg, Germany). Hip and waist circumference were measured with a tape measure to the nearest $0.5 \mathrm{~cm}$. Body surface area (BSA) was calculated using 
Mosteller formula [16]. Body composition was measured by dual X-ray absorptiometry (DXA) using a Lunar Prodigy Advance fan beam scanner (GE Medical Systems, Madison, WI, USA) and analyzed using Prodigy enCORE software version 16.10.15).

Blood pressure (BP) was assessed using three right brachial oscillometric measurements (Omron, M6W BP monitor-device, Omron HealthCare Europe, Hoofddorp, The Netherlands) at rest in the sitting position. The mean of the two lowest readings were used in analyses.

Laboratory tests where assessed from venous blood samples collected between 8 and 10 am following overnight fasting and analyzed according to standard procedures at the Helsinki University Hospital laboratory.

Current medications, diagnoses, and smoking history was assessed by questionnaire. Hypertension was defined as either a systolic blood pressure $>140 \mathrm{mmHg}$, diastolic blood pressure $>90 \mathrm{mmHg}$, or use of anti-hypertensive medication. Diabetes was defined as a fasting glucose $\geq 6.9 \mathrm{mmol} / \mathrm{L}$, glycated hemoglobin $(\mathrm{HbA} 1 \mathrm{c}) \geq 48 \mathrm{mmol} / \mathrm{mol}$, or use of glucose lowering medication. Hypercholesterolemia was defined as LDL $>3.0 \mathrm{mmol} / \mathrm{L}$ or treatment with cholesterol lowering medication. Metabolic syndrome was classified according to AHA guidelines, fulfilling at least three out of following five criteria: (1) Waist circumference $>102 \mathrm{~cm}$ in men and $88 \mathrm{~cm}$ in women; (2) Triglycerides $>1.7 \mathrm{mmol} / \mathrm{L}$ or treatment for hypertriglyceridemia; (3) High-density lipoproteins $<1.03 \mathrm{mmol} / \mathrm{L}$ in men and $1.3 \mathrm{mmol} / \mathrm{L}$ in women; (4) Systolic blood pressure $>130 \mathrm{mmHg}$, diastolic blood pressure $>85 \mathrm{mmHg}$, or antihypertensive medication; and (5) Fasting plasma glucose $>6.1 \mathrm{mmol} / \mathrm{L}$ or glucose lowering medication [17]. Waist circumference was not available for HSCT cohort 2.

\subsection{Vascular Ultrasound}

Vascular VHRU moving cine-clips were acquired from carotid, radial and femoral arteries using Vevo MD (VisualSonics, 2016, Toronto, ON, Canada) equipped with UHF22, UHF48, and UHF70 transducers (center frequencies $15 \mathrm{MHz}, 30 \mathrm{MHz}$, and $50 \mathrm{MHz}$ respectively, cohort 1) or Vevo 770 (VisualSonics, 2005, Toronto, ON, Canada, cohort 2) equipped with 25, 35, and $55 \mathrm{MHz}$ transducers. All images were acquired by one experienced investigator (TS) as previously reported [12]. Previous intra-arterial lines in the vessels scanned were prospectively recorded by interview and inspection of skin scar. Measurements were performed offline with the operator blinded to subject characteristics from the far wall in end-diastole with manual electronic calipers $[12,15]$. The intra-observer coefficients of variations (CV) for different arterial VHRU measurements were 1.2-2.9\% for LD, 6.9-9.8\% for IMT, and $7.6-28.6 \%$ for AT, and inter-observer CVs were $1.5-4.6 \%$ for LD, $6.0-10.4 \%$ for IMT, and $5.9-20.5 \%$ for AT [18].

We have recently histologically validated the far wall four-line pattern in the VHRU arterial image and shown that the intima layer thickness can independently and separately be accurately quantified using the leading edge-to-leading edge technique in arteries with a histological intima layer thickness of more than $0.06 \mathrm{~mm}$ [12]. Due to the low prevalence of intimal thickening in the sample, we recorded intimal thickening as a binominal variable defined as the presence of a four-line pattern in the muscular artery far wall. Cohen's Kappa for intra- and inter-observer agreements were $k=1.00$ and $\kappa=0.85$, respectively.

Carotid artery lumen diameter was further measured during peak systole, and the local carotid artery $\beta$-stiffness index (CBSI) and distensibility coefficient (CDC) were calculated as:

$$
\begin{gathered}
C D C=1000 \times \frac{(C C A L A S-C C A L A D) \div C C A L A D}{(B P S-B P D)} \\
C B S I=\frac{\operatorname{lna}\left(\frac{B P S}{B P D}\right)}{(C C A L D S-C C A L D D) / C C A L D D}
\end{gathered}
$$

where $B P D=$ diastolic blood pressure, $B P S=$ systolic blood pressure, $C C A L A D=$ Carotid artery diastolic lumen area, $C C A L A S=$ carotid artery systolic lumen area, $C C A L D D=$ carotid artery diastolic lumen diameter, and $C C A L D S=$ carotid artery systolic diameter. 


\subsection{Plaque-Screening}

The carotid artery was screened from the common carotid artery prior to the bifurcation, throughout the bulb and the proximal parts of the internal and external carotid arteries. The femoral artery was screened from the common femoral artery throughout the bifurcation and the proximal parts of both the deep and superficial femoral arteries. Plaques were defined in accordance with the Mannheim consensus as a focal thickening of the vascular wall fulfilling at least one of three criteria: (1) maximum IMT $>1.5 \mathrm{~mm}$, (2) IMT increase of $0.5 \mathrm{~mm}$, or (3) $50 \%$ increase in IMT compared with the surrounding vascular wall. Plaque burden was further classified as single or multiple plaques [19]. Cohen's Kappa for intra- and inter-observer agreement on plaque presence were $\mathrm{k}=1.00$ and $\mathrm{k}=0.90$, respectively, and for multiple plaque presence $\mathrm{k}=0.95$ and $\mathrm{k}=0.86$, respectively.

\subsection{Pulse Wave Velocity}

Regional PWV was measured in HSCT cohort 1 by one investigator (TS) using mechanosensors (Complior Analyse, Alam Medical, Saint-Quentin-Fallavier, France) with transit time recorded simultaneously at carotid, radial, and femoral arteries. The direct distance between the recording sites was measured with a tape measure to the nearest $0.1 \mathrm{~cm}$. The direct distance between carotid and femoral sites was multiplied by 0.8 . The $\mathrm{CV}$ for repeat measures were $1.2 \%$ for carotid-radial and $3.6 \%$ for carotid-femoral PWV.

\subsection{Data Analysis}

Data are presented as mean and standard deviation, median and interquartile range, or proportions, as appropriate. Continuous variables were assessed for normal distribution using Shapiro-Wilks test. Group-wise comparisons were done using independent Student's $t$-test for normally distributed variables, Mann-Whitney U-test for non-normally distributed variables, and Fisher's exact test for binominal variables. Prevalence of CVD risk factors and carotid plaques were compared to a population reference using Chi-square goodness of fit.

Group differences between vascular parameters were further compared using ANCOVA adjusting for age and BSA. PWV models were further adjusted for heart rate and mean arterial pressure. Comparison between multiple groups after sample stratification was assessed using Fisher-Freeman-Halton Exact-test, post hoc pair wise Fisher's exact tests with Bonferroni adjusted significance levels for binominal variables, and for continuous variables with ANOVA post hoc Bonferroni adjusted pair wise $t$-tests.

Multiple linear regression and ANCOVA models were used to assess direct effects of treatment, i.e., TBI, CED, and anthracyclines on carotid IMT adjusting for BMI, hypertension, glucose, blood cholesterols, and smoking with non-significant parameters excluded from the final model. Models were built using a bootstrapped sample (1000 repeats) and validated using the study sample. Interaction effects were assessed, as appropriate. Multicollinearity was investigated using variance influence factor (VIF), with VIF not exceeding 2.5 for any parameter in any of the models. Normality and homoscedasticity of residuals was assessed, as appropriate.

Intra- and inter-observer agreements were assessed using CV for continuous variables and Cohen's Kappa $(\kappa)$ for binominal variables.

\section{Results}

\subsection{HSCT Patient and Control Characteristics}

Mean age at diagnosis was 8.1 years (IQR 3.0-12.0 years) and follow-up time was 17.5 years (IQR 14.1-23.0 years). The primary diagnoses were acute lymphoid leukemia $(n=26)$, high-risk neuroblastoma $(n=18)$, severe aplastic anemia $(n=6)$, acute myeloid leukemia $(n=5)$, chronic granulomatous disease $(n=2)$, myelodysplastic syndrome $(n=2)$, high risk retinoblastoma $(n=1)$, dyserythropoietic anemia $(n=1)$, and Chediak-Higashi syndrome $(n=1)$. Forty-six patients 
(74\%) had TBI, out of which $5(8 \%)$ had additional gonadal irradiation and $7(11 \%)$ additional CNS-irradiation. Forty-two $(68 \%)$ patients had cyclophosphamide (median cumulative dose $5541 \mathrm{mg} / \mathrm{m}^{2}$, range $\left.2000-23,183 \mathrm{mg} / \mathrm{m}^{2}\right)$, whereas $41(66 \%)$ had anthracyclines (median cumulative dose among receivers $165 \mathrm{mg} / \mathrm{m}^{2}$, range $40-500 \mathrm{mg} / \mathrm{m}^{2}$ ), with radiated patients receiving more anthracyclines than non-radiated patients. Acute graft-versus-host disease (aGVHD) was recorded in 33 patients $(53 \%)$, with severe GVHD (grade 3-4) in 10 patients (16\%). Chronic GVHD (cGVHD) was recorded and graded in 10 patients $(16 \%)$ at the study visit with five patients showing moderate to severe cGVHD $(8 \%)$. Further background data is presented in Supplementary Table S1.

Data on anthropometrics and CVD risk factors in HSCT survivors and controls at follow-up are presented in Table 1. HSCT survivors were shorter and lighter than controls, and there was no difference in BMI or prevalence of overweight or obesity. There were no statistically significant differences in systolic or diastolic blood pressures between HSCT survivor and control groups, but heart rate was higher among HSCT survivors.

Table 1. Study subject characteristics at follow-up.

\begin{tabular}{|c|c|c|c|c|c|}
\hline & \multicolumn{2}{|c|}{ HSCT $(n=62)$} & \multicolumn{2}{|c|}{ Controls $(n=44)$} & \\
\hline & Mean & SD & Mean & SD & \\
\hline & Median & Q1; Q3 & Median & Q1; Q3 & \\
\hline & $n$ & $\%$ & $n$ & $\%$ & $p$-Value \\
\hline Age [years] & 25.9 & $21.1 ; 30.1$ & 25.2 & $\begin{array}{l}21.6 \\
29.9\end{array}$ & 0.914 \\
\hline Sex [Female] & 11 & $18 \%$ & 11 & $25 \%$ & 0.467 \\
\hline Height $[\mathrm{cm}]$ & 165.5 & 10.8 & 177.6 & 8.8 & $<0.001$ \\
\hline Weight $[\mathrm{kg}]$ & 64.6 & 19.0 & 76.5 & 11.9 & $<0.001$ \\
\hline Body surface area [m2] & 1.71 & 0.30 & 1.94 & 0.19 & $<0.001$ \\
\hline BMI $[\mathrm{kg} / \mathrm{m} 2]$ & 23.1 & 4.9 & 24.2 & 3.2 & 0.208 \\
\hline Waist-hip ratio ${ }^{a}$ & 0.94 & 0.08 & 0.87 & 0.06 & $<0.001$ \\
\hline Heart rate $[1 / \mathrm{min}]$ & 77.3 & 16.5 & 62.0 & 12.1 & $<0.001$ \\
\hline Systolic blood pressure [mmHg] & 124.6 & 13.4 & 120.9 & 10.1 & 0.114 \\
\hline Diastolic blood pressure [mmHg] & 71.1 & 10.1 & 67.8 & 6.7 & 0.047 \\
\hline Overweight (BMI 25+) $[n]$ & 18 & $29 \%$ & 15 & $34 \%$ & 0.671 \\
\hline Obese (BMI 30+) $[n]$ & 7 & $11 \%$ & 2 & $5 \%$ & 0.300 \\
\hline \multicolumn{6}{|l|}{ Cardiovascular disease risk factors } \\
\hline Fat percentage [\%] & 29.3 & 8.7 & - & - & \\
\hline Hypercholesterolemia $[n]$ & 21 & $34 \%$ & 1 & $2 \%$ & $<0.001$ \\
\hline $\mathrm{LDL}[\mathrm{mmol} / \mathrm{L}]$ & 3.22 & 0.89 & - & - & \\
\hline Triglycerides [mmol/L] & 1.28 & $0.86 ; 2.00$ & - & - & \\
\hline hsCRP $[\mathrm{mg} / \mathrm{L}]$ & 1.55 & $0.72 ; 3.77$ & - & - & \\
\hline Hypertension $[n]$ & 21 & $34 \%$ & & & \\
\hline Diabetes $[n]$ & 5 & $8 \%$ & 0 & $0 \%$ & 0.075 \\
\hline $\mathrm{HbA} 1 \mathrm{c}[\mathrm{mmol} / \mathrm{mol}]$ & 35 & $32 ; 38$ & - & - & \\
\hline Metabolic syndrome $[n]^{\mathrm{a}}$ & 9 & $21 \%$ & 0 & $0 \%$ & 0.021 \\
\hline Blood pressure medication $[n]$ & 9 & $15 \%$ & 0 & $0 \%$ & 0.010 \\
\hline Statin medication $[n]$ & 6 & $10 \%$ & 0 & $0 \%$ & 0.040 \\
\hline Previous or current smoking $[n]$ & 20 & $32 \%$ & 0 & $0 \%$ & $<0.001$ \\
\hline Current Smoking $[n]$ & 10 & $16 \%$ & 0 & $0 \%$ & 0.005 \\
\hline
\end{tabular}

Anthropometric measures and cardiovascular disease risk factors among study subjects. Laboratory tests are not available for controls. ${ }^{a}$ Data available for HSCT cohort 1 only $(n=43)$. BMI, Body mass index, LDL, Low-density lipoprotein, HbA1c, Glycated Hemoglobin.

Prevalence of overweight among HSCT patients was lower compared to a Finnish reference population ( 29.0 vs. $58.8 \%, p=0.001$ ), whereas the difference in the prevalence of obesity was borderline insignificant (11.3 vs. $21.5 \%, p=0.051$ ) [20]. Compared to a Finnish reference population of similar age, HSCT patients had a higher prevalence of hypertension ( 33.9 vs. $12.8 \%, p<0.001$ ), similar prevalence 
of hypercholesterolemia ( 33.9 vs. $28.6 \%, p=0.353)$, and a higher prevalence of diabetes ( 8.1 vs. $2.3 \%$, $p=0.002)[21]$.

The prevalence of carotid artery plaques was significantly higher among HSCT patients than in a previously reported Norwegian population of similar age (24.2 vs. $2.8 \%, p<0.001)$ [21]. The presence of arterial intimal thickening was also significantly more prevalent among HSCT patients than we have previously reported among subjects aged $25-50$ years with significant CVR burden ( 31.0 vs. $4.5 \%$, $p<0.001)[12]$.

\subsection{Arterial CVD Surrogate Marker Differences between HSCT Survivors and Controls}

Sample images of arterial plaques and arterial wall layers among HSCT survivors are illustrated in Figure 1.

Unadjusted and adjusted vascular dimension data are presented in Table 2. HSCT survivors had smaller lumen diameters in radial and femoral arteries compared to controls and thicker IMT in carotid, brachial, and femoral arteries, with no consistent difference in adventitia thickness. The difference in LD disappeared in all arteries except for the femoral arteries when adjusting for age and BSA, whereas the difference in IMT between HSCT survivors and controls was exaggerated and statistically significant for all evaluated arteries, including the radial artery.

The increase in CIMT with age was steeper in independent linear regression models among HSCT survivors ( $\beta=8.8 \mu \mathrm{m} /$ year, CI95\% 5.1-12.5 $\mu \mathrm{m} /$ year) compared to controls ( $\beta=3.4 \mu \mathrm{m} /$ year, CI95\% 1.0-5.7 $\mu \mathrm{m} /$ year), seen as a significant interaction term in a multiple linear regression model (age * HSCT $\beta=5.5 \mu \mathrm{m} /$ year CI95\% 0.9-10.1 $\mu \mathrm{m} /$ year, $p=0.020$, Figure 2, Supplementary Table S2).

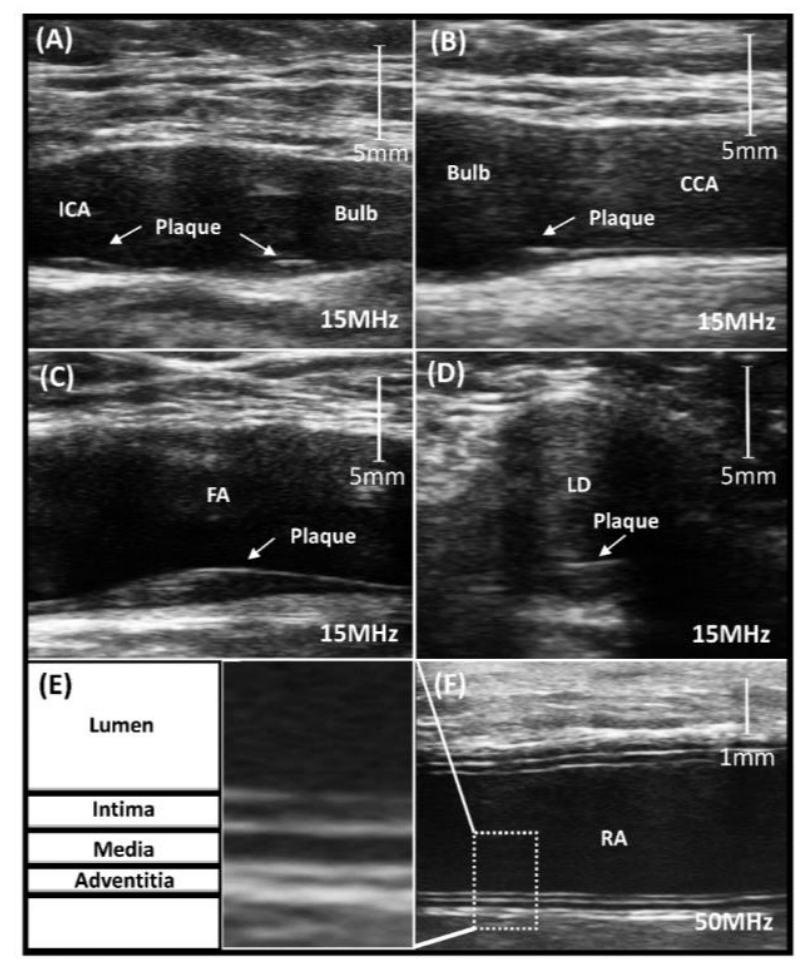

Figure 1. Ultrasound images of bulb and ICA (A), and proximal bulb (B) of a carotid artery with two plaques $(15 \mathrm{MHz})$. Ultrasound images of a femoral artery with a large hypoechoic dorsal plaque in (C) longitudinal and (D) transverse imaging planes (15 MHz). Ultrasound image of a radial artery (F) with zoomed far wall (E) with diffuse intimal thickening seen as a four-line pattern (50 MHz). CCA, common carotid artery; FA, femoral artery; ICA, internal carotid artery; LD, Lumen. 


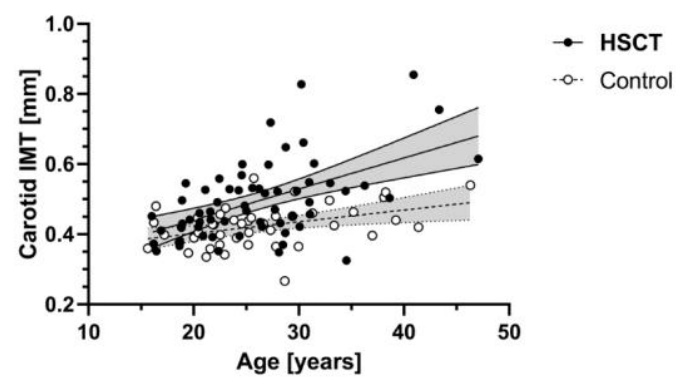

Figure 2. Scatter plots of (A) linear regressions (CI 95\% shaded area) of carotid intima-media thickness (CIMT) increase with age among HSCT and controls showing the significant increase in the age-related increase of CIMT in HSCT patients compared to controls.

Table 2. Comparison of vascular parameters between HSCT and controls.

\begin{tabular}{|c|c|c|c|c|c|c|c|}
\hline & \multicolumn{4}{|c|}{ Unadjusted } & \multirow{2}{*}{\multicolumn{3}{|c|}{$\begin{array}{c}\text { Adjusted for Age and BSA } \\
\text { HSCT-Control }\end{array}$}} \\
\hline & \multicolumn{2}{|l|}{ HSCT } & \multicolumn{2}{|l|}{ Control } & & & \\
\hline & Mean & SD & Mean & SD & $p$-Value & $\Delta$ Mean & CI95\% \\
\hline & $n=62$ & & $n=44$ & & & & \\
\hline Common carotid artery & & & & & & & \\
\hline $\mathrm{LD}[\mathrm{mm}]$ & 5.39 & 0.55 & 5.47 & 0.50 & 0.410 & 0.13 & $-0.04 ; 0.31$ \\
\hline IMT [mm] & 0.49 & 0.11 & 0.42 & 0.06 & $<0.001$ & $0.07^{b}$ & $0.04 ; 0.11$ \\
\hline Radial artery & & & & & & & \\
\hline $\mathrm{LD}[\mathrm{mm}]$ & 1.85 & 0.37 & 1.96 & 0.36 & 0.119 & 0.05 & $-0.08 ; 0.19$ \\
\hline IMT [mm] & 0.16 & 0.03 & 0.16 & 0.03 & 0.355 & $0.014^{\mathrm{a}}$ & $0.00 ; 0.03$ \\
\hline $\mathrm{AT}[\mathrm{mm}]$ & 0.07 & 0.02 & 0.07 & 0.03 & 0.597 & 0.00 & $-0.01 ; 0.01$ \\
\hline Brachial artery & & & & & & & \\
\hline $\mathrm{LD}[\mathrm{mm}]$ & 3.47 & 0.74 & 3.63 & 0.61 & 0.224 & 0.16 & $-0.05 ; 0.36$ \\
\hline IMT [mm] & 0.18 & 0.05 & 0.16 & 0.03 & 0.006 & $0.03^{b}$ & $0.02 ; 0.05$ \\
\hline AT $[\mathrm{mm}]$ & 0.13 & 0.03 & 0.12 & 0.02 & 0.275 & 0.01 & $0.00 ; 0.02$ \\
\hline Femoral artery & & & & & & & \\
\hline $\mathrm{LD}[\mathrm{mm}]$ & 6.71 & 1.01 & 7.84 & 1.00 & $<0.001$ & $-0.65^{\mathrm{a}}$ & $-1.02 ;-0.28$ \\
\hline IMT [mm] & 0.37 & 0.09 & 0.33 & 0.07 & 0.021 & $0.04^{\mathrm{a}}$ & $0.01 ; 0.07$ \\
\hline AT $[\mathrm{mm}]$ & 0.26 & 0.08 & 0.25 & 0.05 & 0.177 & 0.01 & $-0.02 ; 0.04$ \\
\hline Intimal thickening & $n$ & $\%$ & $n$ & $\%$ & $p$-value & & \\
\hline Any arteries $[n]$ & 18 & $31 \%$ & 1 & $2 \%$ & $<0.001$ & - & - \\
\hline Femoral artery $[n]$ & 8 & $14 \%$ & 0 & $0 \%$ & 0.019 & - & - \\
\hline Radial artery $[n]$ & 9 & $15 \%$ & 1 & $2 \%$ & 0.042 & - & - \\
\hline Plaques & $n$ & $\%$ & $n$ & $\%$ & $p$-value & & \\
\hline Any plaques $[n]$ & 18 & $30 \%$ & 2 & $5 \%$ & 0.001 & - & - \\
\hline Carotid Plaque $[n]$ & 15 & $24 \%$ & 2 & $5 \%$ & 0.007 & - & - \\
\hline Femoral Plaque $[n]$ & 8 & $13 \%$ & 0 & $0 \%$ & 0.020 & - & - \\
\hline Arterial stiffness & Mean & SD & Mean & $\mathrm{SD}$ & $p$-value & $\Delta$ Mean & $\mathrm{CI} 95 \%$ \\
\hline CBSI & 5.5 & 1.7 & 4.4 & 1.4 & $<0.001$ & $1.45^{\mathrm{c}}$ & $0.85 ; 2.05$ \\
\hline $\mathrm{CDC}[\% / 10 \mathrm{mmHg}]$ & 4.4 & 1.5 & 5.9 & 1.9 & $<0.001$ & $-1.78^{c}$ & $-2.38 ;-1.19$ \\
\hline Carotid-femoral PWV [m/s] & 8.7 & 1.4 & 8.5 & 1.4 & 0.656 & 0.05 & $-0.68 ; 0.78$ \\
\hline Carotid-radial PWV $[\mathrm{m} / \mathrm{s}]$ & 9.5 & 1.9 & 9 & 1.5 & 0.286 & 0.03 & $-1.02 ; 1.07$ \\
\hline
\end{tabular}

Three HSCT survivors and one control were excluded from the intimal-thickening analysis due to a history of previous intra-arterial catheters. Intimal thickening in any artery was more frequent 
among HSCT than controls (18/62 vs. 1/44, $p<0.001$; Supplementary Table S3) with no significant difference between non-radiated and radiated survivors ( $4 / 15$ vs. $14 / 43, p=0.756)$. HSCT survivors with intimal thickening were significantly older than HSCT without intimal thickening $(29.5 \pm 8.0$ years vs. $24.7 \pm 5.6$ years, $p=0.01$, Supplementary Figure S1A).

Carotid and femoral plaques were more frequent among HSCT than controls (18/62 vs. 2/44, $p=0.001$; Supplementary Table S2). Twelve out of 15 survivors with plaques had multiple plaques, whereas none of the controls had multiple plaques $(p=0.005)$.

Carotid beta stiffness index (CBSI) was higher and carotid distensibility coefficient (CDC) lower in HSCT compared to controls (adjusted $p<0.001$ ). However, there was no statistically significant differences between HSCT survivors and controls in carotid-radial or carotid-femoral PWVs (Table 2).

\subsection{Arterial CVD Surrogate Markers in Relation to Treatment Regimens and CVD Risk Factors among HSCT}

There were no significant differences in arterial wall dimensions, PWV, intimal thickening, or arterial plaques in HSCT exposed to TBI compared with non-exposed (Supplementary Tables S3 and S4).

HSCT survivors with local additional boost radiation therapy of the gonads had higher prevalence of femoral artery plaques compared to HSCT survivors treated with TBI only ( $3 / 5$ vs. $4 / 41 p=0.020$ ) as well as compared to non-radiated subjects TBI ( $3 / 5$ vs. $1 / 16, p=0.028$, Supplementary Table S3). Local additional radiation therapy of the CNS area was not, however, associated with more carotid plaques (1/7 vs.13/39 $p=0.413$, Supplementary Table S3). Patients with plaques were slightly older than patients without plaques ( 30.0 years vs. 24.5 years, $p=0.003$ ), but plaque presence was unrelated to traditional CVD risk factors in our sample (results not shown).

There were minor differences between allogenic and autologous HSCT survivors (Supplementary Table S5), but only femoral IMT remained statistically significantly thicker in allogenic HSCT after adjusting for age, BSA, and sex. Local carotid artery stiffness measures were higher in allogenic HSCT.

In ANCOVA models, age, BMI, HbA1c, hypertension, hs-CRP, and cumulative anthracycline dose were all independently related with CIMT among HSCT recipients (Table 3). There was also a dose related association between CIMT and cumulative anthracycline exposure. Similarly, there was a positive trend between CIMT and cumulative number of CVD risk factor and metabolic syndrome criteria (Figure 3A,B). No difference was, however, seen between HSCT without metabolic or CVD risk factors and healthy controls.

Table 3. ANCOVA model predicting carotid intima-media thickness among HSCT.

\begin{tabular}{|c|c|c|c|}
\hline Dependent Variable & $\mathbf{n}$ & $\mathbf{R}^{2}$ & Model $p$-Value \\
\hline Carotid intima-media thickness $[\mu \mathrm{m}]$ & 62 & 0.597 & $<0.001$ \\
\hline Independent variables & $\beta$ & C195\% & $p$-value \\
\hline Constant & 176.8 & $79.6 ; 274.0$ & 0.584 \\
\hline Age [years] & 8.5 & $5.3 ; 11.8$ & $<0.001$ \\
\hline \multicolumn{4}{|l|}{ BMI $\left[\mathrm{kg} / \mathrm{m}^{2}\right]$} \\
\hline$<25$ & - & - & - \\
\hline $25-30$ & -15.1 & $-70.1 ; 39.9$ & 0.584 \\
\hline$>30$ & 85.2 & $14.2 ; 156.1$ & 0.020 \\
\hline $\mathrm{HbA} 1 \mathrm{c}>42 \mathrm{mmol} / \mathrm{mol}[0=$ no, $1=$ yes $]$ & 86.6 & $24.3 ; 148.8$ & 0.007 \\
\hline Hypertension $[0=$ no, $1=$ yes $]$ & 50.5 & $1.6 ; 99.4$ & 0.043 \\
\hline $\mathrm{hsCRP}>2.5 \mathrm{mg} / \mathrm{L}$ & 56.3 & $11.9 ; 100.7$ & 0.014 \\
\hline \multicolumn{4}{|l|}{ Anthracycline cum. dose/BSA $\left[\mathrm{mg} / \mathrm{m}^{2}\right]$} \\
\hline 0. No Anthracyclines & - & - & - \\
\hline 1. $0-150 \mathrm{mg}$ & 55.38 & $-1.5 ; 112.2$ & 0.056 \\
\hline 2. $>150 \mathrm{mg}$ & 80.0 & $28.2 ; 131.7$ & 0.003 \\
\hline
\end{tabular}

ANCOVA-model predicting carotid intima-media thickness among HSCT. Inclusion of LDL/HDL, triglycerides, smoking, total body irradiation, or exposure to cyclophosphamides did not affect the fit of the model. BMI, Body mass index; HbA1c, Glycated Hemoglobin; hsCRP, high sensitivity C-reactive protein; C195, 95\% confidence interval. 
Both CBSI and CDC were significantly predicted by age, low-density lipoprotein, and TBI exposure (Supplementary Table S6), but no association with PWV was found. Radial, brachial, and femoral artery IMT were predicted by age but not by CVD risk factors or treatment regimens (results not shown). There were no statistically significant associations between vascular parameters and previous aGVHD, aGVHD grade, or active cGVHD or cGVHD severity (results not shown).

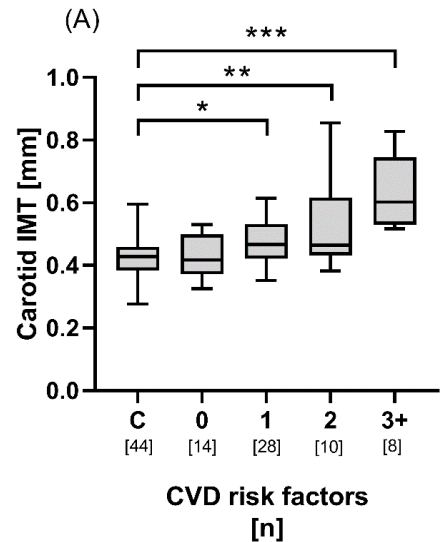

[n]

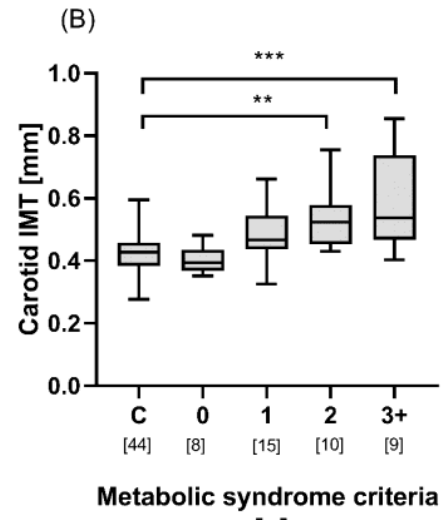

[n]

Figure 3. Comparison of carotid artery intima-media thickness (IMT) among controls (C, $n=44$ ) and HSCT ( $n=60$ ) with (A) 0, 1, 2, and 3+ of the following cumulative number of cardiovascular disease (CVD) risk factors: hypertension (systolic blood pressure $>130 \mathrm{mmHg}$, diastolic blood pressure $>85 \mathrm{mmHg}$, or antihypertensive medication), $\mathrm{HbA} 1 \mathrm{c}>42 \mathrm{mmol} / \mathrm{mol}, \mathrm{BMI}>30 \mathrm{~g} / \mathrm{m}^{2}$, anthracycline cumulative exposure $>150 \mathrm{mg} / \mathrm{m}^{2}$, and hsCRP $>2.5 \mathrm{mg} / \mathrm{L}$ and (B) $0,1,2$, and $3+$ of the following cumulative number of American Heart Association guideline metabolic syndrome criteria (out of 5) in HSCT cohort $1(n=42)$ : high waist circumference ( $>102 \mathrm{~cm}$ in men and $88 \mathrm{~cm}$ in women); high plasma triglycerides $(>1.7 \mathrm{mmol} / \mathrm{L})$; or treatment for hypertriglyceridemia, low plasma high-density lipoproteins ( $<1.03 \mathrm{mmol} / \mathrm{L}$ in men and $<1.3 \mathrm{mmol} / \mathrm{L}$ in women), hypertension (systolic blood pressure $>130 \mathrm{mmHg}$, diastolic blood pressure $>85 \mathrm{mmHg}$, or antihypertensive medication), and high fasting plasma glucose ( $>6.1 \mathrm{mmol} / \mathrm{L}$ or glucose lowering medication). ${ }^{*} p<0.05 ;{ }^{* *} p<0.01 ;{ }^{* * *} p<0.001$.

\section{Discussion}

We compared arterial wall morphology and stiffness in a cohort of young adult HSCT survivors of childhood malignancies and hematological diseases with healthy controls and further assessed relations between vascular parameters, treatment regimens, and CVD risk factors. We report, similar to previous reports, reduced body size, higher heart rate, and increased prevalence of subclinical arterial plaques among HSCT long-term survivors [3,4,22]. Overall, among HSCT patients, CVD risk factors were more common than in the average population, and arterial walls were thicker, stiffer, and presented more plaques and intimal thickening compared to healthy controls without CVD risk factors, consistent with early vascular ageing $[13,23]$.

Local arterial stiffening and plaques were related with radiation therapy, and femoral artery plaques were pronounced in patients with additional gonadal irradiation, as previously reported [24-26]. A similar relationship was not seen between additional CNS irradiation and carotid artery plaque likely explained by the use of protective shielding of the neck region [27]. Plaques were present over the whole age spectrum in our patients, starting from $<20$ years, an age group normally not burdened by plaques $[21,28,29]$. This indicates early toxic arterial plaque formation and local arterial stiffening as a sequel of radiation therapy in childhood. There was also an increased prevalence of arterial intimal thickening identified in subjects $<20$ years of age. We have previously reported ultrasound derived arterial intimal thickening among subjects burdened by CVD risk factors starting from age 36 years [12]. Allogenic HSCT survivors showed increased local carotid artery stiffness and increased femoral IMT compared with autologous HSCT survivors. 
This study further shows increased prevalence and clustering of CVD risk factors among HSCT survivors compared with the Finnish population of similar age. CIMT has previously been shown to be related with hsCRP, whereas in our sample CIMT was further significantly predicted by cumulative burden of CVD risk factors and treatment with anthracyclines [30,31]. Anthracyclines have previously been linked to adverse cardiac effects, e.g., cardiomyopathies, but preclinical and clinical pilots studies suggest an adverse effect of anthracyclines on arterial endothelial function [32,33]. Confounding mechanisms related with disease, disease severity and different treatment protocols could, however, potentially explain the dose dependence reported in the present study between anthracycline and CIMT.

The Increased CIMT was, contrary to our expectations, not related to TBI exposure, but related mainly with CVD risk factors and metabolic syndrome criteria among HSCT survivors. Importantly, we found no difference between CIMT of healthy controls and HSCT survivors without CVD risk factors. We further showed a significantly steeper increase in CIMT with age among HSCT survivors compared with controls, consistent with CVD related accelerated early arterial ageing among HSCT patients. In combination, these observations suggest that the early prevention of CVD risk factors could potentially prevent cardiovascular disease progression among HSCT patients.

The main limitation of this study is the heterogeneity in diseases and treatment regimes in our relatively small sample including a low number of females that limits a more in-depth stratified analysis evaluating associations related with sex, diseases, treatments or doses, and vascular outcomes. A further major limitation is the differences in CVD risk factor profiles between HSCT patients and our healthy control group without CVD risk factors as our controls serve as a healthy vascular reference. This limits the conclusions that can be drawn from the results. By comparing the prevalence of CVD risk factors with population data, our study setting allows us to conclude that there is a high prevalence of CVD risk factors among the HSCT patients. We can further conclude that HSCT patients have an adverse vascular profile compared to healthy controls as well as with previously reported young adult average Nordic populations [20,21], and we conclude that among HSCT patients the adverse vascular profile is related partly to CVD risk factors and partly to treatment regimes. We can, however, not answer whether the effect of CVD risk factors on the vascular profile is higher among HSCT patients than in a young adult population with a similarly high CVD risk factor distribution. A further limitation is the relatively large drop-out rate in cohort 1 , which could predispose to selection bias. There was, however, no significant difference in background factors between participants and non-participants. Significant strengths include a relatively long follow-up time and well characterized background and clinical data as well as the use of recently developed and validated novel methods to assess subclinical changes of the arterial wall. The follow-up time was, however, not long enough for the development of cardiovascular disease events.

In conclusion, we report significant adverse alterations in vascular morphology and function consistent with early arterial ageing in young adult survivors following childhood HSCT. Although plaque formation and arterial stiffening were mainly related with radiation therapy, we found that intima-media thickness was mainly related with clustering of CVD risk factors. This suggests that vascular changes develop through different pathways among HSCT survivors including a direct toxic damage of radiation leading to plaque formation, while CVD risk factor clustering plays a key role in premature and early intima-media layer thickening. The results suggest that early interventions to modify CVD risk factors potentially could improve long-term cardiovascular outcomes in this high-risk population.

Supplementary Materials: The following are available online at http:/www.mdpi.com/2077-0383/9/7/2208/ s1, Figure S1: Scatter plots of carotid intima-media thickness among HSCT and controls, Table S1: Clinical characteristics of hematopoietic stem cell transplantation (HSCT) survivors; Table S2. ANCOVA-model assessing age-related increase of carotid intima-media thickness in HSCT survivors and controls; Table S3. Comparison of plaque and intimal thickening stratified for radiation therapy exposure; Table S4. Comparison of vascular parameters between TBI-exposed and unexposed HSCT survivors; Table S5. Comparison of vascular parameters between autologous HSCT and allogenic HSCT survivors; Table S6. ANCOVA-models predicting measures of arterial stiffness among HSCT. 
Author Contributions: J.K.M.S. was responsible for data analysis and writing of the manuscript. T.S. was responsible for all study visits, data collection, and analysis of vascular data and supervised data analysis and writing of the manuscript. A.S. recruited cohort 2 and was responsible for data management and primary analysis of this cohort. K.J. designed the studies and coordinated the follow-up visits. T.S. and K.J. are guarantors of the study. All authors have read and agreed to the published version of the manuscript.

Funding: This study has been supported by grants from the Sigrid Juselius Foundation, The Medical Society of Finland, and Finnish Foundation for Pediatric Research, Perklen foundation, Medicinska understödsföreningen Liv och Hälsa, and the Stockmann Foundation, the Swedish Research Council, the Swedish Barncancerfonden, Stockholm County Council Research Funds, the Finnish Cancer Society, Väre Foundation for Pediatric Cancer Research.

Conflicts of Interest: The authors have no conflicts of interest to declare.

\section{Abbreviations}

$\begin{array}{ll}\text { AT } & \text { Adventitia thickness } \\ \text { BSA } & \text { Body surface area } \\ \text { BMI } & \text { Body mass index } \\ \text { BP } & \text { Blood pressure } \\ \text { BPD } & \text { Diastolic blood pressure } \\ \text { BPS } & \text { Systolic blood pressure } \\ \text { CBSI } & \text { Carotid } \beta \text {-stiffness index } \\ \text { CDC } & \text { Carotid distensibility coefficient } \\ \text { CED } & \text { Cyclophosphamide equivalent dose } \\ \text { CIMT } & \text { Carotid intima-media thickness } \\ \text { CNS } & \text { Central nervous system } \\ \text { CV } & \text { Coefficient of variation } \\ \text { CVD } & \text { Cardiovascular disease } \\ \text { GVHD } & \text { Graft versus host disease } \\ \text { HbA1c } & \text { Glycated hemoglobin } \\ \text { hsCRP } & \text { High sensitivity C-reactive protein } \\ \text { HSCT } & \text { Hematopoietic stem cell transplantation } \\ \text { IMT } & \text { Intima-media thickness } \\ \text { PWV } & \text { Pulse wave velocity } \\ \text { SD } & \text { Standard deviation } \\ \text { TBI } & \text { Total body irradiation } \\ \text { VHRU } & \text { Very-high resolution ultrasound } \\ & \end{array}$

\section{References}

1. Coebergh, J.W.; Reedijk, A.M.; de Vries, E.; Martos, C.; Jakab, Z.; Steliarova-Foucher, E.; Kamps, W.A. Leukaemia incidence and survival in children and adolescents in Europe during 1978-1997. Report from the Automated Childhood Cancer Information System project. Eur. J. Cancer 2006, 42, 2019-2036. [CrossRef]

2. Chow, E.J.; Mueller, B.A.; Baker, K.S.; Cushing-Haugen, K.L.; Flowers, M.E.; Martin, P.J.; Friedman, D.L.; Lee, S.J. Cardiovascular hospitalizations and mortality among recipients of hematopoietic stem cell transplantation. Ann. Intern. Med. 2011, 155, 21-32. [CrossRef]

3. Gurney, J.G.; Ness, K.K.; Sibley, S.D.; O’Leary, M.; Dengel, D.R.; Lee, J.M.; Youngren, N.M.; Glasser, S.P.; Baker, K.S. Metabolic syndrome and growth hormone deficiency in adult survivors of childhood acute lymphoblastic leukemia. Cancer 2006, 107, 1303-1312. [CrossRef] [PubMed]

4. Perkins, J.L.; Kunin-Batson, A.S.; Youngren, N.M.; Ness, K.K.; Ulrich, K.J.; Hansen, M.J.; Petryk, A.; Steinberger, J.; Anderson, F.S.; Baker, K.S. Long-term follow-up of children who underwent hematopoeitic cell transplant (HCT) for AML or ALL at less than 3 years of age. Pediatr. Blood Cancer. 2007, 49, 958-963. [CrossRef] [PubMed]

5. Talvensaari, K.K.; Lanning, M.; Tapanainen, P.; Knip, M. Long-term survivors of childhood cancer have an increased risk of manifesting the metabolic syndrome. J. Clin. Endocrinol. Metab. 1996, 81, 3051-3055. [PubMed] 
6. Khanna, A.; Pequeno, P.; Gupta, S.; Thavendiranathan, P.; Lee, D.S.; Abdel-Qadir, H.; Nathan, P.C. Increased Risk of All Cardiovascular Disease Subtypes Among Childhood Cancer Survivors: Population-Based Matched Cohort Study. Circulation 2019, 140, 1041-1043. [CrossRef] [PubMed]

7. Mulrooney, D.A.; Hyun, G.; Ness, K.K.; Ehrhardt, M.J.; Yasui, Y.; Duprez, D.; Howell, R.M.; Leisenring, W.M.; Constine, L.S.; Tonorezos, E.; et al. Major cardiac events for adult survivors of childhood cancer diagnosed between 1970 and 1999: Report from the Childhood Cancer Survivor Study cohort. BMJ 2020, 368, 16794. [CrossRef]

8. Gavriilaki, E.; Gkaliagkousi, E.; Sakellari, I.; Anyfanti, P.; Douma, S.; Anagnostopoulos, A. Early Prediction of Cardiovascular Risk after Hematopoietic Cell Transplantation: Are We There Yet? Biol. Blood Marrow Transplant. 2019, 25, e310-e316. [CrossRef]

9. Sarkola, T.; Redington, A.; Keeley, F.; Bradley, T.; Jaeggi, E. Transcutaneous very-high-resolution ultrasound to quantify arterial wall layers of muscular and elastic arteries: Validation of method. Atherosclerosis 2010, 212, 516-523. [CrossRef]

10. Eklund, C.; Friberg, P.; Gan, L.M. High-resolution radial artery intima-media thickness and cardiovascular risk factors in patients with suspected coronary artery disease-Comparison with common carotid artery intima-media thickness. Atherosclerosis 2012, 221, 118-123. [CrossRef]

11. Eklund, C.; Omerovic, E.; Haraldsson, I.; Friberg, P.; Gan, L.M. Radial artery intima-media thickness predicts major cardiovascular events in patients with suspected coronary artery disease. Eur. Heart J. Cardiovasc. Imaging 2014, 15, 769-775. [CrossRef] [PubMed]

12. Sundholm, J.K.M.; Paetau, A.; Alback, A.; Pettersson, T.; Sarkola, T. Non-Invasive Vascular Very-High Resolution Ultrasound to Quantify Artery Intima Layer Thickness: Validation of the Four-Line Pattern. Ultrasound Med. Biol. 2019, 45, 2010-2018. [CrossRef] [PubMed]

13. Vatanen, A.; Sarkola, T.; Ojala, T.H.; Turanlahti, M.; Jahnukainen, T.; Saarinen-Pihkala, U.M.; Jahnukainen, K. Radiotherapy-related arterial intima thickening and plaque formation in childhood cancer survivors detected with very-high resolution ultrasound during young adulthood. Pediatr. Blood Cancer 2015, 62, 2000-2006. [CrossRef] [PubMed]

14. Green, D.M.; Nolan, V.G.; Goodman, P.J.; Whitton, J.A.; Srivastava, D.; Leisenring, W.M.; Neglia, J.P.; Sklar, C.A.; Kaste, S.C.; Hudson, M.M.; et al. The cyclophosphamide equivalent dose as an approach for quantifying alkylating agent exposure: A report from the Childhood Cancer Survivor Study. Pediatr. Blood Cancer 2014, 61, 53-67. [CrossRef]

15. Jagasia, M.H.; Greinix, H.T.; Arora, M.; Williams, K.M.; Wolff, D.; Cowen, E.W.; Palmer, J.; Weisdorf, D.; Treister, N.S.; Cheng, G.S.; et al. National Institutes of Health Consensus Development Project on Criteria for Clinical Trials in Chronic Graft-versus-Host Disease: I. The 2014 Diagnosis and Staging Working Group report. Biol. Blood Marrow Transpl. 2015, 21, 389-401. [CrossRef] [PubMed]

16. Mosteller, R.D. Simplified calculation of body-surface area. N. Engl. J. Med. 1987, 317, 1098.

17. Grundy, S.M.; Cleeman, J.I.; Daniels, S.R.; Donato, K.A.; Eckel, R.H.; Franklin, B.A.; Gordon, D.J.; Krauss, R.M.; Savage, P.J.; Smith, S.C., Jr.; et al. American Heart Association; National Heart, Lung, and Blood Institute Diagnosis and management of the metabolic syndrome: An American Heart Association/National Heart, Lung, and Blood Institute Scientific Statement. Circulation 2005, 112, 2735-2752. [CrossRef]

18. Sundholm, J.; Gustavsson, T.; Sarkola, T. Semi-automatic border detection software for the quantification of arterial lumen, intima-media and adventitia layer thickness with very-high resolution ultrasound. Atherosclerosis 2014, 234, 283-287. [CrossRef]

19. Touboul, P.J.; Hennerici, M.G.; Meairs, S.; Adams, H.; Amarenco, P.; Bornstein, N.; Csiba, L.; Desvarieux, M.; Ebrahim, S.; Hernandez Hernandez, R.; et al. Mannheim Carotid Intima-Media Thickness and Plaque Consensus (2004-2006-2011). Cerebrovasc. Dis. 2012, 34, 290-296. [CrossRef]

20. Koponen, P.; Borodulin, K.; Lundqvist, A.; Sääksjärvi, K.; Koskinen, S. Health, Functional Capacity and Welfare in Finland-FinHealth 2017 Study; Report 4/2018; National Institute for Health and Welfare (THL): Helsinki, Finland, 2018.

21. Joakimsen, O.; Bonaa, K.H.; Stensland-Bugge, E.; Jacobsen, B.K. Age and sex differences in the distribution and ultrasound morphology of carotid atherosclerosis: The Tromso Study. Arterioscler. Thromb. Vasc. Biol. 1999, 19, 3007-3013. [CrossRef] 
22. Freycon, F.; Casagranda, L.; Trombert-Paviot, B. The impact of severe late-effects after 12 Gy fractionated total body irradiation and allogeneic stem cell transplantation for childhood leukemia (1988-2010). Pediatr. Hematol. Oncol. 2019, 36, 86-102. [CrossRef] [PubMed]

23. Dengel, D.R.; Kelly, A.S.; Zhang, L.; Wang, Q.; Hodges, J.S.; Steinberger, J.; Baker, K.S. Vascular Structure and Function in Cancer Survivors after Hematopoietic Stem Cell Transplantation. Biol. Blood Marrow Transplant. 2019, 25, 151-156. [CrossRef] [PubMed]

24. Jurado, J.A.; Bashir, R.; Burket, M.W. Radiation-induced peripheral artery disease. Catheter. Cardiovasc. Interv. 2008, 72, 563-568. [CrossRef] [PubMed]

25. Katras, T.; Baltazar, U.; Colvett, K.; Rush, D.; Dunn, J.; Stanton, P., Jr. Radiation-related arterial disease. Am. Surg. 1999, 65, 1176-1179. [PubMed]

26. Lawson, J.A. Surgical treatment of radiation induced atherosclerotic disease of the iliac and femoral arteries. J. Cardiovasc. Surg. 1985, 26, 151-156.

27. Patil, V.M.; Oinam, A.S.; Chakraborty, S.; Ghoshal, S.; Sharma, S.C. Shielding in whole brain irradiation in the multileaf collimator era: Dosimetric evaluation of coverage using SFOP guidelines against in-house guidelines. J. Cancer. Res. Ther. 2010, 6, 152-158. [CrossRef]

28. Salonen, R.; Seppanen, K.; Rauramaa, R.; Salonen, J.T. Prevalence of carotid atherosclerosis and serum cholesterol levels in eastern Finland. Arteriosclerosis 1988, 8, 788-792. [CrossRef]

29. Sturlaugsdottir, R.; Aspelund, T.; Bjornsdottir, G.; Sigurdsson, S.; Thorsson, B.; Eiriksdottir, G.; Gudnason, V. Prevalence and determinants of carotid plaque in the cross-sectional REFINE-Reykjavik study. BMJ Open 2016, 6, e012457. [CrossRef]

30. Vatanen, A.; Hou, M.; Huang, T.; Soder, O.; Jahnukainen, T.; Kurimo, M.; Ojala, T.H.; Sarkola, T.; Turanlahti, M.; Saarinen-Pihkala, U.M.; et al. Clinical and biological markers of premature aging after autologous SCT in childhood cancer. Bone Marrow Transpl. 2017, 52, 600-605. [CrossRef]

31. Wilhelmsson, M.; Glosli, H.; Ifversen, M.; Abrahamsson, J.; Winiarski, J.; Jahnukainen, K.; Hasle, H. Nordic Society of Pediatric Hematology and Oncology (NOPHO) Long-term health outcomes in survivors of childhood AML treated with allogeneic HSCT: A NOPHO-AML Study. Bone Marrow Transpl. 2019, 54, 726. [CrossRef]

32. Murata, T.; Yamawaki, H.; Yoshimoto, R.; Hori, M.; Sato, K.; Ozaki, H.; Karaki, H. Chronic effect of doxorubicin on vascular endothelium assessed by organ culture study. Life Sci. 2001, 69, 2001-2695. [CrossRef]

33. Chow, Y.; Chin, C.; Dahl, G.; Rosenthal, D.N. Anthracyclines Cause Endothelial Injury in Pediatric Cancer Patients: A Pilot Study. J. Clin. Oncol. 2006, 24, 925-928. [CrossRef] [PubMed] 